Article

\title{
Systematic Analysis and Functional Validation of Citrus Pectin Acetylesterases (CsPAEs) Reveals that CsPAE2 Negatively Regulates Citrus Bacterial Canker Development
}

\author{
Qiang Li ${ }^{1}, * \mathbb{}$, Jia Fu ${ }^{1}$, Xiujuan Qin ${ }^{1}$, Wen Yang ${ }^{1}$, Jingjing $\mathrm{Qi}^{1}{ }^{1}$, Zhengguo Li ${ }^{2}$, Shanchun Chen ${ }^{1}$ \\ and Yongrui $\mathrm{He}^{1,2, *}$ \\ 1 Citrus Research Institute, Southwest University/Chinese Academy of Agricultural Sciences, \\ Chongqing 400712, China; fjduolaimi@126.com (J.F.); qinxiujuan-cric@foxmail.com (X.Q.); \\ yangwen114429@foxmail.com (W.Y.); qijingjing-cric@foxmail.com (J.Q.); citgvd@cric.cn (S.C.) \\ 2 Key Laboratory of Plant Hormones and Development Regulation of Chongqing, School of Life Sciences, \\ Chongqing University, Chongqing 401331, China; zhengguoli@cqu.edu.cn \\ * Correspondence: liqiang@cric.cn (Q.L.); heyongrui@cric.cn (Y.H.)
}

Received: 9 November 2020; Accepted: 9 December 2020; Published: 11 December 2020

\begin{abstract}
The present study was designed to serve as a comprehensive analysis of Citrus sinensis (C. sinensis) pectin acetylesterases (CsPAEs), and to assess the roles of these PAEs involved in the development of citrus bacterial canker (CBC) caused by Xanthomonas citri subsp. citri (Xcc) infection. A total of six CsPAEs were identified in the genome of C. sinensis, with these genes being unevenly distributed across chromosomes 3, 6, and 9, and the unassembled scaffolds. A subset of CsPAEs were found to be involved in responses to Xcc infection. In particular, CsPAE2 was identified to be associated with such infections, as it was upregulated in CBC-susceptible variety Wanjincheng and inversely in CBC-resistant variety Calamondin. Transgenic citrus plants overexpressing CsPAE2 were found to be more susceptible to $\mathrm{CBC}$, whereas the silencing of this gene was sufficient to confer $\mathrm{CBC}$ resistance. Together, these findings provide evolutionary insights into and functional information about the CsPAE family. This study also suggests that CSPAE2 is a potential candidate gene that negatively contributes to bacterial canker disease and can be used to breed $C B C$-resistant citrus plants.
\end{abstract}

Keywords: pectin acetylesterase (PAE); Xanthomonas citri subsp. citri (Xcc); citrus bacterial canker (CBC); Citrus sinensis

\section{Introduction}

All living beings must be able to efficiently and effectively detect and respond to danger [1] The primary plant cell wall functions as a major protective barrier that can prevent pathogenic infection [2,3]. These cell walls are composed of heavily cross-linked polysaccharide polymer networks [2,4-6], with pectin, cellulose, and hemicellulose fibrils forming a matrix that serves as a barrier that can only be penetrated via mechanical force or the secretion of specific digestive enzymes [3]. In addition to its barrier function, the cell wall is essential for plant cells to detect and respond to biotic stress. Multiple different receptors and other sensory molecules are present within the plasma membranes of plant cells, allowing for the detection of apoplastic infections and the resultant induction of appropriate symplastic immune responses [7]. A number of different molecules are capable of binding wall-associated receptors in order to induce such immune responses, including cell wall-derived molecules, DNA fragments, and misfolded proteins [8]. Many of these elicitor compounds 
are derived from pectin, which is composed of a rhamnogalacturonan or homogalacturonan backbone and is the most prevalent polysaccharide within the cell wall of nongraminaceous plants [9].

Pectin composes up to a third of the cell wall by mas, and can be modified via C2 and/or C3 galacturonic acid residue acetylation [10]. The specific acetylation and methylation patterns present on pectin fragments ultimately determine the degree to which they function as elicitors of immune responses, and pectin acetylesterase (EC 3.1.1.6, PAE) can cleave pectin acetylester bonds to modulate these patterns [10-13]. Pectin de-esterification can result in acetate and/or methanol release, allowing these compounds to be readily incorporated back into metabolic pathways within the plant. This can also result in the accumulation of negatively charged carboxyl groups, potentially leading to a drop in $\mathrm{pH}$ that may impact the activity of various apoplastic proteins and ion channels, including wall-loosening expansins [14,15]. Pectin de-esterification also impacts the apoplastic reactive oxygen species' (ROS) homeostasis, which has not been sufficiently studied [16]. Multiple independent reports have found that pectin configurations are key determinants of the ability of plants to muster effective immune responses against pathogens, and as such there is clear value in further studying related regulatory pathways in an effort to identify novel disease management strategies [17]. At present, however, only a limited number of studies have explored plant cell wall-mediated immune responses at the metabolic and transcriptional levels, and these studies have primarily focused on such responses in the context of pectin demethylation.

In the CAZy database, plant PAEs are members of the CE13 carbohydrate esterase family [18]. Recent improvements in plant genomic datasets have led to more widespread PAE annotation and study in different plant species. However, few studies have explored PAE physiological functions to date, or the evolution, function, and structure of the PAE gene family in plants $[13,19,20]$. These studies give the insight that a lower plant has fewer pectin-related gene family members compared to Arabidopsis, and only one ancestral PAE in the earliest land plant $[11,20,21]$. In addition, there have not been sufficient studies of the function and expression of PAEs in different plants, and the roles in plant development of these proteins remain uncertain. The overexpression of PtPAE2 in tobacco has been shown to have a significant adverse impact on floral development, leading to decreased pollen formation and resultant sterility [12]. In addition, plants bearing AtPAE 8 and AtPAE9 mutations have been found to exhibit short inflorescence stems [19].

Past studies demonstrated that PAEs likely play important regulatory roles in plant responses to biotic stressors. For example, AtPAE2 and AtPAE4 were upregulated in response to biotic stressors, suggesting they may be key regulators of plant defense responses [11]. In response to pathogenic infection and other stressors, the cell wall undergoes a number of morphological and physiological changes regulated by expansins (EXPs) [22], PAEs [11], and xyloglucan endotransglucosylase/hydrolases (XTHs) to product oligogalacturonides (OGAs), which are fragments of the homogalacturonan domains of pectin $[2,23]$. The OGAs released by the cell wall can function as signaling intermediates, modulating ROS homeostasis to activate plant immune responses [24-27]. These OGAs are damage-associated molecular patterns (DAMPs) [28], and their accumulation can induce microbial resistance in Arabidopsis and tobacco [29]. In wheat, infection level of Blumeria graminis could be induced by acetylated OGAs and non-acetylated OGAs, which provides evidence for elicitation and protection effects of preventive treatments with OGAs in wheat and for new properties of acetylated OGAs [24]. More research into the role of pectin acetyl esterification in plant immunity is, however, still needed [3].

Citrus bacterial canker (CBC) caused by Xanthomonas citri subsp. citri $(\mathrm{Xcc})$ is a serious bacterial disease [30-33]. In the present study, we conducted the comprehensive in silico annotation of C. sinensis PAEs [34,35]. We further conducted a functional analysis of PAE genes and explored their relevance to $\mathrm{CBC}$ resistance. The functions involved in $\mathrm{CBC}$ development were then validated by reverse genetics strategies. Together, our findings highlight novel potential approaches to reducing the $\mathrm{CBC}$ susceptibility. 


\section{Results}

\subsection{Six CsPAEs Were Identified and Annotated in C. sinensis Genome}

Through exhaustive data mining and annotation efforts, we were able to identify and characterize six CSPAE genes named CsPAE1-6 (Table 1). Of these genes, all six were predicted based upon Citrus annotation project (CAP) database, and five were also predicted by Phytozome. In order to validate these putative CsPAEs, the best expressed sequence tag (EST) hits were extracted from the EST dataset (NCBI), confirming that all six of these CsPAEs were identified with a total of 21 ESTs (Table S1). Among the six, CsPAE1 possesses the most ESTs (12). The PAE genes of Wanjincheng were cloned and sequenced according to the PAEs in the reference genome. We finally found that only CsPAE4 contained a 2-base difference from the reference gene in CAP (CAP ID: Cs6g06280.1). The gene, CsPAE2 coding sequences (CDS), and protein sequences of CsPAEs are included in Table S2. The isoelectric point (PI), means of annotation, and molecular weight (MW) are compiled in Table 1. The CsPAE genes encodes 386 (CsPAE2) to 423 (CsPAE6) amino acid residues with MW 42542.05-49247.08 Dalton. CsPAE2 and CsPAE4 contain more acidic amino acids, making the proteins appear acidic (PI $<7)$, while more basic amino acids made CsPAE1, 3, 5, and 6 appear basic (PI $>7)$.

Table 1. List and details of PAEs identified in C. sinensis genome.

\begin{tabular}{ccccccc}
\hline Name & CAP ID & AA NO. & MW (DD) & PI & EST NO. & Annotation \\
\hline CsPAE1 & Cs3g10410.1 & 399 & $43,822.24$ & 8.68 & 12 & CAP, P, EST \\
CsPAE2 & Cs3g10420.1 & 386 & $42,542.05$ & 5.86 & 1 & CAP, P, EST \\
CsPAE3 & Cs6g01740.1 & 423 & $47,321.84$ & 9.01 & 3 & CAP, EST \\
CsPAE4 & Cs6g06280.1 & 424 & $47,569.82$ & 6.41 & 1 & CAP, P, EST \\
CsPAE5 & Cs9g17480.1 & 397 & $44,834.28$ & 8.39 & 2 & CAP, P, EST \\
CsPAE6 & orange1.1t01789.1 & 441 & $49,247.08$ & 8.24 & 2 & CAP, P, EST \\
\hline
\end{tabular}

All PAEs are listed. MW: molecular weight. AA: amino acid. PI: isoelectric point. In annotation: P: Prediction by phytozome; CAP: citrus annotation project (CAP) prediction. EST: genes with EST hits.

\subsection{Phylogenetic Analysis of CsPAEs}

In order to study the phylogenetic relationships of PAEs between organisms, phylogeny of CsPAEs were conducted based upon comparing their full amino acid sequences to those of AtPAEs. The resultant ML phylogenetic tree indicated that CsPAEs can be separated into three distinct clades (clades 1-3) in accordance with the clades used for AtPAE identification (Figure 1) [11]. Specifically, CsPAE3 and CsPAE6 were in clade 1, CsPAE4 and CsPAE5 were in clade 2, and CsPAE1 and CsPAE2 were in clade 3. Based on the phylogeny, genes in pairs CsPAE5-AtPAE9, CsPAE4-AtPAE4/5, and CsPAE3-AtPAE3/6 displayed close relationships that indicate the interspecific homologies. The phylogenetic tree also showed the intraspecific homology between citrus and Arabidopsis. Four pairs of homologous PAEs (AtPAE3 and AtPAE6, AtPAE10 and AtPAE12, AtPAE4 and AtPAE5, AtPAE7 and AtPAE11) were detected in Arabidopsis, whereas only one pair (CsPAE1 and CsPAE2) was in citrus.

\subsection{Conserved Domains and Secondary Structures of CsPAEs}

All six CsPAEs were predicted to contain an N-terminal signal peptide and a PAE domain (Pfam: PF03283) (Figure 2), and $9 \alpha$-helices and $14 \beta$-strands in their secondary structures. Eleven conserved motifs were detected in the PAE domain of CsPAEs, including catalytic active site $S, D$, and $H$ residues consistent with strong catalytic site conservation [11]. Indeed, conserved GCSxG, NxayDxxQ, and HCQ motifs were present within both CsPAEs and AtPAEs. Furthermore, these PAEs contain four cysteine residues that facilitate disulfide bond formation and enhance enzymatic thermostability (Figure 2). 


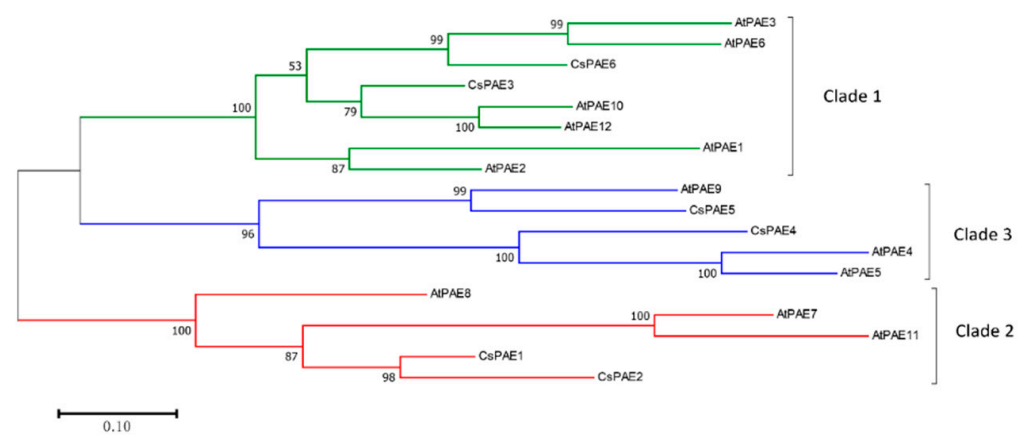

Figure 1. Maximum-Likelihood (ML) phylogeny of all the PAEs from C. sinensis and A. thaliana. An ML tree was constructed based on the PAE amino acid sequences of $A$. thaliana and C. sinensis (12 and 6 , respectively) with MEGA V7.0 (bootstrap $=500$, Poisson model). Branches are drawn to scale, with length corresponding to the number of substitutions per site. Sub-family assignments are shown on the right. Clades are color-coded as indicated.
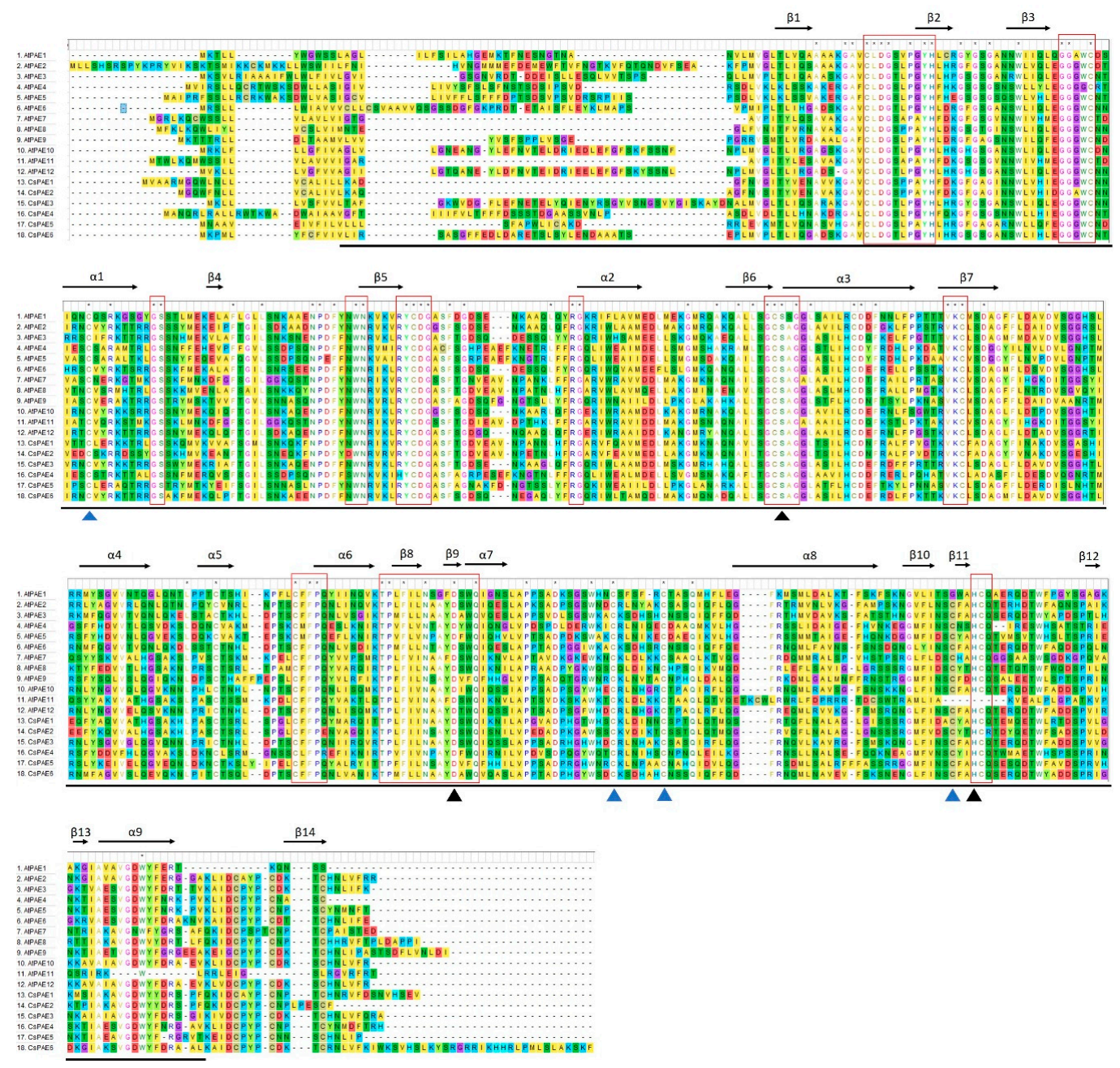

Figure 2. Alignment of the protein sequences of CsPAEs and AtPAEs. The Muscle tool in MEGA V7.0 was used for the alignment of full-length $A$. thaliana and $C$. sinensis PAEs. Red rectangles were used to highlight conserved PAE motifs. Secondary structural elements are shown by black arrows along the top of the protein sequences, with $\alpha$ and $\beta$ corresponding to $\alpha$-helices and $\beta$-strands, respectively. The $\mathrm{S}, \mathrm{D}$, and $\mathrm{H}$ active catalytic sites are marked using black triangles, while cysteine residues capable of disulfide bond formation are marked by blue triangles. Consensus sequences are marked an asterisks over the corresponding residues. 


\subsection{Physical Distributions and Gene Structures of CSPAE Genes}

The six CSPAE genes identified were located on three chromosomes (CHR3, 6, and 9) and the unassembled scaffolds. The exon-intron structures of CsPAEs were similar to those of AtPAEs with respect to the presence of many (10-12) introns [11]. CSPAE1-5 were found to contain 11 introns, while CsPAE6 contained 12 (Figure 3). Combining the chromosomal localization and the phylogeny (Figure 1), we conclude that CSPAE1 and CsPAE2 have suffered tandem duplication events in the evolution process, leading to the birth of a gene and neofunctionalization [36]. These two evolved into genes containing different CDSs (similarity: 82\%), opposite acid/base preferences (basic vs. acidic), and significantly different intron sequences (Figure 3). The "newborn" CsPAE2 could possess new functions in citrus.

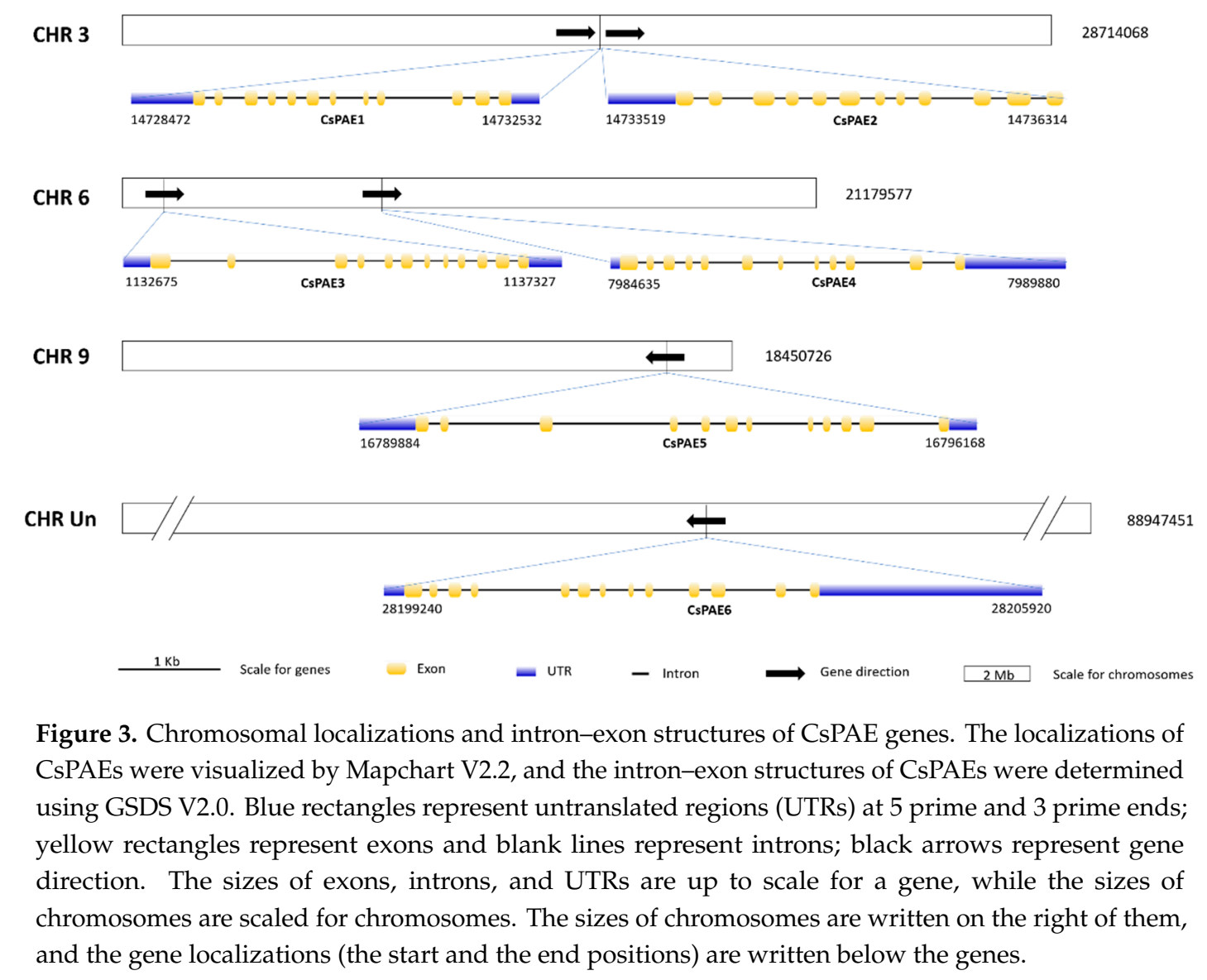

\subsection{CsPAE2 Was Inversely Induced by Xcc in CBC-Susceptible and CBC-Resistant Varieties}

We next explored the functional roles of CSPAEs in response to biotic stress by assessing CSPAE expression patterns in leaves that had been infected by Xcc within $48 \mathrm{~h}$ post inoculation (hpi) by qRT-PCR. Specifically, we found that CSPAE2, CSPAE3, and CSPAE5 were upregulated in the CBC-susceptible variety Wanjincheng. Of these genes, we found that CSPAE2 was downregulated in the CBC-resistant variety Calamondin, whereas CSPAE3 and CSPAE5 were still upregulated in response to Xcc infection in Calamondin (Figure 4). This suggested that CSPAE2 may be a potential susceptibility-related gene that plays a role in responding to Xcc infection. No significant changes in the expression of the other three CSPAEs were detected in response to Xcc infection in either Wanjincheng or Calamondin. Based on these results, we therefore selected CSPAE2 as a potential candidate gene worthy of further study by reverse genetics strategies. 

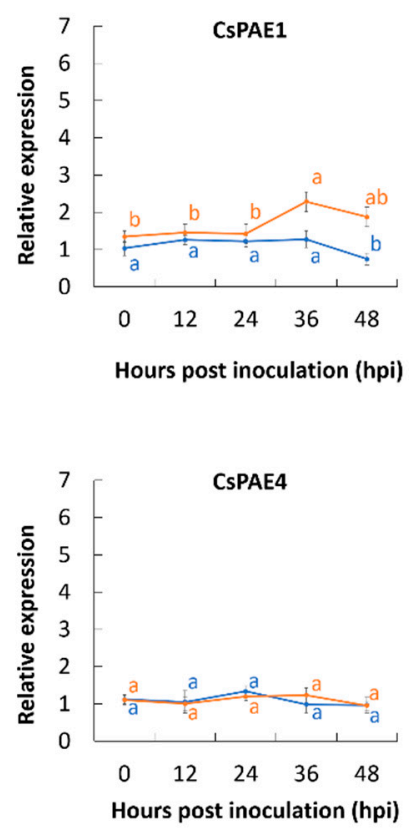

- Calamondin $\left(\mathrm{CBC}^{R}\right)$
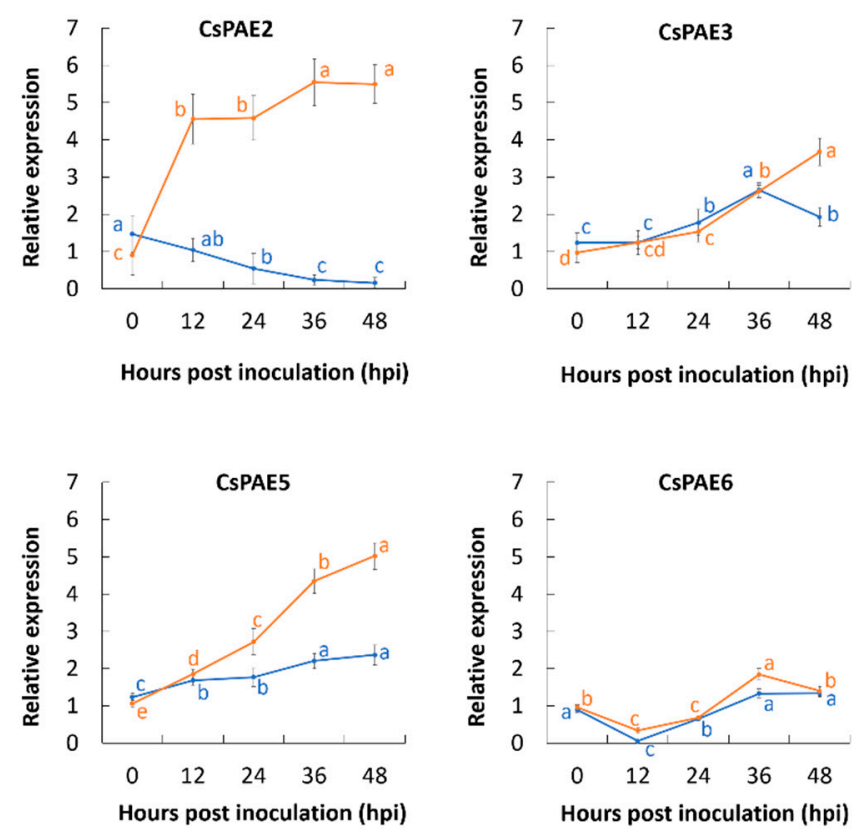

Figure 4. CSPAE expression profiles under the infection of Xcc. Wanjincheng (orange) and Calamondin (blue) were infected with Xcc for 0, 12, 24, 36, and $48 \mathrm{~h}$, after which CsPAE expression was assessed via qRT-PCR, with CsActin being used for the normalization. Uninfected control samples were inoculated using LB medium. Data are means \pm SEs. Duncan's multiple range test was used to compare the data $(p=0.05)$, with three biological replicates per sample. The significance of the difference was marked by lowercase letters (a-e).

\subsection{Overexpression of CsPAE2 Confers CBC Susceptibility}

In order to explore the role of CsPAE2 in the context of CBC, we next generated transgenic citrus plants overexpressing this protein using a CSPAE2 overexpression plasmid that contained a glucuronidase (GUS) coding sequence under the control of a CaMV 35S promoter (Figure 5A). We confirmed the successful integration of CsPAE2 in three overexpression plants (labeled OE1, OE2, and OE3) via both PCR and GUS assays (Figure 5B,C). These transgenic plants exhibited growth rates comparable to those of wild type plant (WT), but exhibited more bifurcation compared to WT plant (Figure 5D). When we assessed these three plants via qRT-PCR, we were able to confirm that they expressed significantly elevated CSPAE2 levels (29-fold, 36-fold, and 26-fold of WT, respectively) (Figure 5E). The acupuncture method is used to assess and compare the resistance between OE plants and WT [2,37-39]. We found that these OE plants exhibited much larger lesions and more significant symptoms relative to WT (Figure 5F). Disease aggravation was most pronounced in OE2 plants, followed by OE1 and OE3, respectively. In OE2 plants, at 10 days post inoculation (dpi), lesions were approximately $127 \%$ the size of those in WT plants on average (Figure 5G). In addition, transgenic plants exhibited increases in disease severity (DS) by $16 \%(\mathrm{OE} 3)$ to $19 \%$ (OE2) relative to WT (Figure 5H). These results led us to conclude that CsPAE2 overexpression was sufficient to increase CBC susceptibility in transgenic citrus plants. 
A

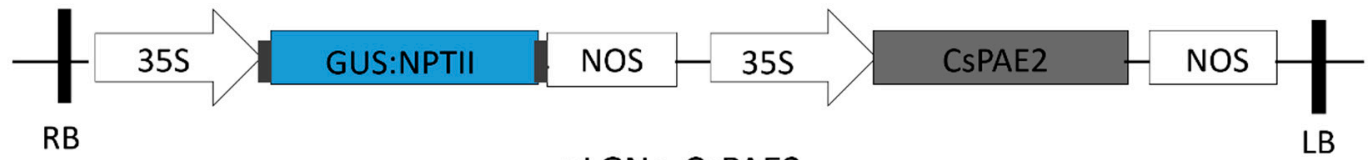

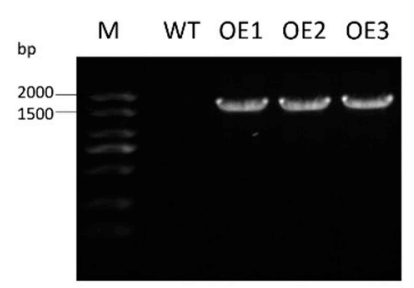

F

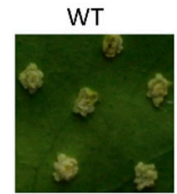

OE2

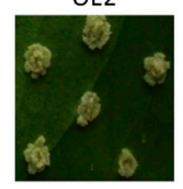

$12 \mathrm{~mm}$
pLGNe-CSPAE2

C

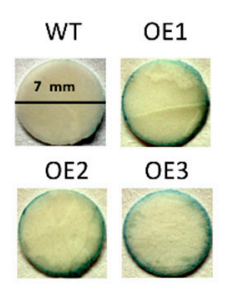

D

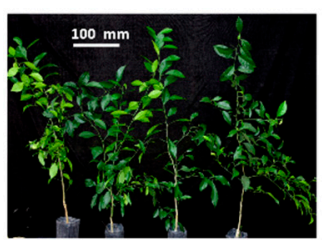

WT OE1 OE2 OE3

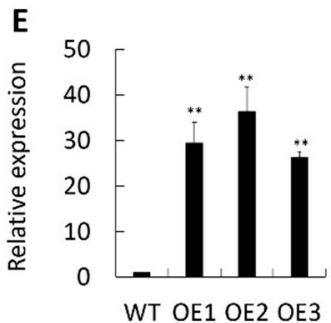

H

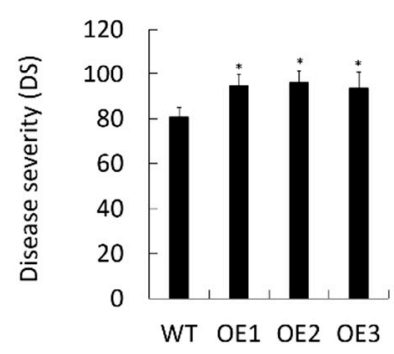

Figure 5. Assessment of Xcc responses in CSPAE2 overexpression plants and WT plant. (A) Plasmid pLGNe-CsPAE2 used for overexpression assay. NPTII, NptII gene; NOS, Nos terminator; GUS, glucuronidase; LB: left border; RB: right border. (B) PCR-mediated validation of transgenic plants. (C) GUS staining-mediated validation of transgenic plants. (D) Phenotypes of the transgenic plants. Scale bar $=100 \mathrm{~mm}$. (E) The expression of CSPAE2 in the indicated plants was measured via qRT-PCR, with CsActin for the normalization. At $10 \mathrm{dpi}$, disease symptoms on the leaves of WT and transgenic plants inoculated with Xcc were assessed (F); lesion size (LS) (G) and disease severity (DS) (H) were assessed. In $(\mathbf{F})$, the scale bar $=12 \mathrm{~mm}$. WT plants served as controls for statistical testing. Data are means \pm SEs $(n \geq 3)$. Duncan's multiple range test was used to compare data of OEs and WT, with three biological replicates per sample $\left({ }^{*} p<0.05 ;{ }^{* *} p<0.01\right)$.

\subsection{CsPAE2 Silencing Increases CBC Resistance}

In order to expand upon the above results, we next knocked down CsPAE2 via RNAi using appropriate constructs inserted into the pLGNe vector (Figure 6A). Three transgenic plants were obtained by PCR (R1, R2, and R3) (Figure 6B) and GUS staining (Figure 6C). Relative to WT, these transgenic plants exhibited higher growth rates (Figure 6D). Expression of CsPAE2 in these plants was significantly reduced to $40 \%, 18 \%$, and $22 \%$ of WT, respectively (Figure 6E). Upon infection with Xcc, the three mutants exhibited smaller pustules than those evident on WT plant (Figure 6F). We were therefore able to conclude that CsPAE2 knockdown can significantly increase Xcc resistance in C. sinensis. Consistent with this, we observed significantly smaller lesion sizes (LS) in these three silenced plants $(75 \%, 63 \%$, and $71 \%$ of WT, respectively) (Figure 6G). Furthermore, an assessment of CBC severity indicated that these three transgenic plants exhibited markedly increased disease severity relative to WT plants (Figure 6H), with consequent decreases in DS of 26\% (R1) and 35\% (R3). These findings therefore confirmed that the knockdown of CSPAE2 is sufficient to confer CBC resistance, thereby-together with the overexpression assay-indicating that CSPAE2 is a CBC susceptibility gene. 
A

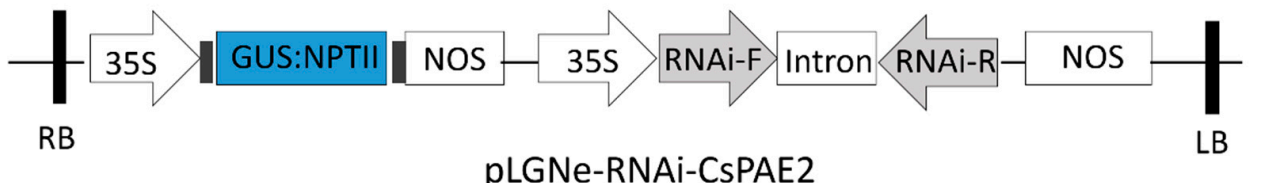

B

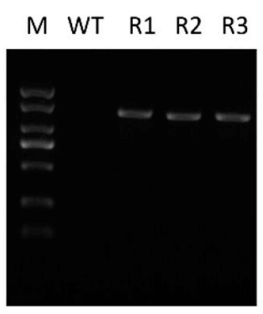

C
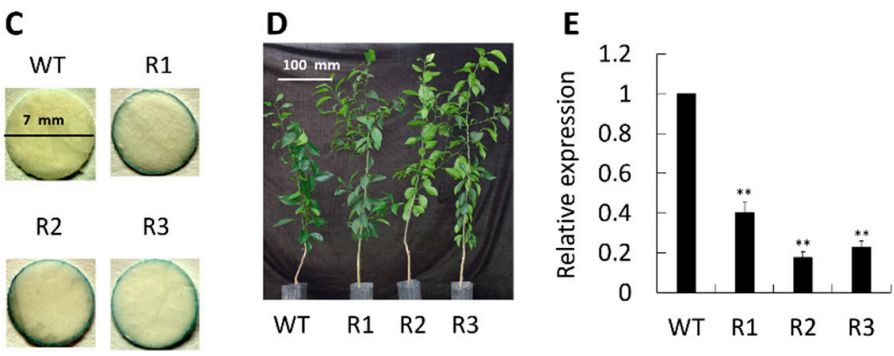

$\mathbf{F}$
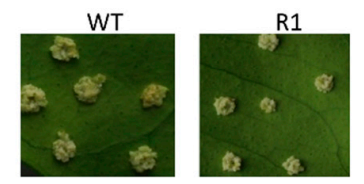

$\mathbf{G}$

H
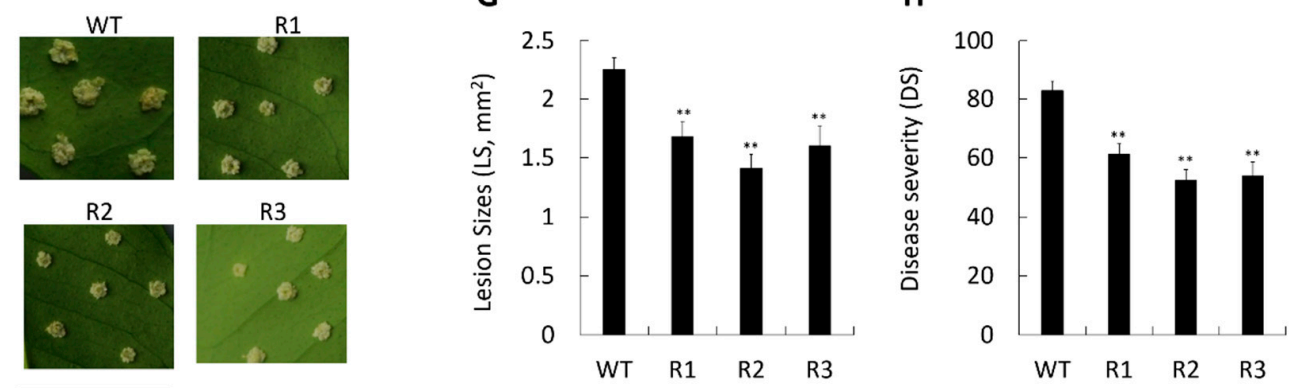

Figure 6. Assessment of Xcc responses in CSPAE2 knockdown plants and WT plant. (A) Plasmid pLGNe-CSPAE2-RNAi used for RNAi assay. NPTII, NptII gene; NOS, Nos terminator; GUS, glucuronidase; LB: left border; RB: right border. (B) PCR-mediated validation of transgenic plants. (C) GUS staining-mediated validation of transgenic plants. (D) The phenotypes of transgenic plants. Scale bar $=100 \mathrm{~mm}$. (E) The expression of CSPAE2 in the indicated plants was measured via qRT-PCR, with CsActin used for the normalization. At $10 \mathrm{dpi}$, disease symptoms on the leaves of WT and transgenic plants inoculated with Xcc were assessed (F), and lesion size (LS) (G) and disease severity (DS) (H) were assessed. In (F), the scale bar $=12 \mathrm{~mm}$. WT plants served as controls for statistical testing. Data are means \pm SEs $(n \geq 3)$. Duncan's multiple range test was used to compare data of OEs and WT, with three biological replicates per sample $(* * p<0.01)$.

\section{Discussion}

In the present study, we first employed a bioinformatics approach in order to comprehensively identify PAEs within the $C$. sinensis genome and to characterize their structures and gene expression profiles. We then further explored the functional relevance of these identified CsPAEs in response to $X c c$ infection, offering novel insights into the role of this gene family in the context of $C B C$ resistance. This is the first study to our knowledge that has explored this topic.

PAEs compose a multi-gene family in higher plants, whereas in lower plants there is only one PAE gene copy [21]. This difference may be related to the differences in the acetylation modifications produced by these different enzymes. Lower plants may exhibit lower levels of acetylation, thereby necessitating reduced PAE enzymatic activity, whereas in higher plants pectin de-acetylation is a more complex process requiring lots of PAEs. We were able to identify in total six PAEs encoded in the $C$. sinensis genome, which is half the number detected in the Arabidopsis genome [11]. The difference in gene family sizes between the two species is related to the number of duplication events. In fact, five duplication events were detected in Arabidopsis, whereas only one was detected in C. sinensis [11]. We then used the sequences of these proteins to construct a phylogenetic tree, grouping these CsPAEs into three clades containing two CsPAEs per clade. Much like PAEs identified 
in Arabidopsis, we found that CsPAEs exhibited high numbers of introns (Figure 3). Intron-containing genes are known to increase their transcription more efficiently than non-intronic genes. These genes can also function as negative regulators of gene expression via generating intronic microRNAs capable of controlling PAE expression profiles in specific tissues or other regulatory contexts [40].

Several studies have shown that PAEs can regulate plant stress responses [11,12,19]. In Arabidopsis, the mutants of putative pectin acetyltransferase genes $P M R 5$ and $P M R 6$ are more susceptible to $B$. cinerea, whereas PMR mutants are less susceptible to powdery mildew infection [25]. CsPAE expression patterns may offer functional insights into their diverse roles in plants. As such, it is possible to better understand the role of CSPAEs in the context of plant defenses by quantifying changes in their expression in response to biotic stressors. In this study, we investigated $C B C$-responsive CSPAE genes via qRT-PCR, revealing CSPAE2, CSPAE3, and CSPAE5 to all exhibit Xcc-dependent changes in their expression levels (Figure 4). Of these genes, we found that CsPAE2 exhibited opposing expression patterns in Calamondin and Wanjincheng, being down-regulated in the former and upregulated in the latter upon Xcc inoculation (Figure 4). Using reverse genetic engineering strategies (overexpression and RNAi silence), we were then further able to determine that CSPAE2 is a potential CBC susceptibility gene (Figures 5 and 6). Regarding the phenotype changes, OE plants exhibited comparable growth rates, and RNAi plants exhibited faster growth rates compared to those of WT plants. Additionally, both the OE plants and RNAi plants possessed more bifurcation compared to WT plants (Figures 5D and 6D). This result suggests that CSPAE2 might also be involved in citrus growth regulation.

This study highlighted the role of CSPAE genes in CBC development, thereby extending the current list of such CBC-related genes. However, many questions relating to this topic remain to be answered. For example, the mechanistic basis for Xcc-mediated induction of CsPAE2 expression remains to be established, as does the functional role of CSPAE2 during CBC infection. The observed differences in CSPAE2 expression in Wanjincheng and Calamondin may provide some insights into the different cis-regulatory elements controlling its upregulation in these species [41]. Many future studies of how PAEs function in the context of plant immune responses are needed, and additional molecular and physiological research regarding the role of CSPAE2 in CBC susceptibility are necessary in order to more fully understand the role of this gene involved in $\mathrm{CBC}$ development.

As such, these findings provide evolutionary insights into and functional investigations of the CsPAE gene family. This study also suggests that CSPAE2 is a potential CBC susceptibility gene that negatively regulates $\mathrm{CBC}$ development and can be used to breed $\mathrm{CBC}$-resistant citrus plants.

\section{Materials and Methods}

\subsection{Annotation and Bioinformatics Analysis of CsPAEs}

The C. sinensis genome and proteome were downloaded from CAP (http://citrus.hzau.edu.cn/ orange) [34,35] and Phytozome (https://phytozome.jgi.doe.gov) [42,43]. A three-step semi-automated process was used to identify and annotate PAEs based on an initial query with $12 \mathrm{~A}$. thaliana PAEs [36,44]. Functional and structural annotation were conducted using HMMER V3.3 (http://www.hmmer. org) [45,46], SMART (https://smart.embl.de) [47], and Gbrowse in CitGVD (http://citgvd.cric.cn) [48]. The identified $C$. sinensis PAEs were termed as CsPAE, and were numbered in the chromosomal order. Muscle was used for protein sequence alignment [49], and MEGA V7.0 was used for the maximum likelihood (ML) phylogenetic assay. The intron-exon structures and chromosomal localizations of CSPAE genes were visualized by Gene Structure Display Server V2.0 (GSDS, http://gsds.gao-lab.org) [50] and Mapchart V2.2 [51] respectively. Signal peptide and subcellular localization predictive analyses were done with SignalP V4.0 (http://www.cbs.dtu.dk/services/SignalP) [52] and CELLO V2.5 (http: //cello.life.nctu.edu.tw) [53] respectively. 


\subsection{Plants and Bacteria}

The National Citrus Germplasm Repository (Chongqing, China) was the source of the plants used in this study. Xcc assays were conducted using the Calamondin (Citrus madurensis) (CBC ${ }^{\mathrm{R}}$ ) and Wanjincheng (Citrus sinensis) $\left(\mathrm{CBC}^{\mathrm{S}}\right)$ varieties, with the latter additionally being used for gene transformation. All plants were grown at $28{ }^{\circ} \mathrm{C}$ in a greenhouse. The Xcc YN1 strain was isolated from naturally infected sweet orange leaves, and was cultured at $28^{\circ} \mathrm{C}$ using peptone-yeast extract-malt extract (PYM).

\subsection{Xcc Assays}

Xcc inducible expression patterns of CsPAEs were measured as in prior reports [39,54]. Briefly, the mature new leaves (approximately 3-month-old leaves) of Calamondin and Wanjincheng (roughly 10-year-old plants) were picked and placed in the culture plates, while keeping the petioles wrapped in cotton that was soaked in $\mathrm{dd}_{2} \mathrm{O}$. The leaves of these plants were then inoculated with 1000-fold dilution of XccYN1 (OD600 $=0.5$, which is equivalent to $5 \times 10^{8} \mathrm{CFU} \cdot \mathrm{mL}^{-1}$ ) and then were incubated at $28^{\circ} \mathrm{C}$ with a $16 \mathrm{~h} \mathrm{light} / 8 \mathrm{~h}$ dark photoperiod. Every $12 \mathrm{hpi}$, samples were collected for analysis through 48 hpi. Samples treated with LB medium was used as the control (CK). Primers are compiled in Table S3.

\subsection{Plant Transformation}

Overexpression plasmids were generated via initially amplifying the full-length CsPAE2 coding sequences (CDS) using the following primers: $\mathrm{F}_{\mathrm{OEC}}$ (CGGGATCCATGGGCCAATGGTTCAATCTTTTA), $\mathrm{R}_{\mathrm{OEC}}$ (CGGAATTCTCAAAAGCAACTCTCTGGCAATGGGT). The PCR product was inserted into the vector $p G L N e$. Silencing vectors were constructed via amplifying a 300-bp fragment using the following primers: F-RIc (GCTCTAGAGGCGCGCCAATGAGCAGAAATTTAACCCA), R-RIc (CGGGATCCATTTAAATGCCAGCATCTGCAAAGCATTT). The amplified product was then inserted into the PUC-RANi so as to yield an RNAi sequence that was obtained and cloned into the pLGNe vector. Transformation of Wanjincheng shoot segments was conducted using Agrobacterium tumefaciens containing appropriate plasmids, as previously described [38,55].

\subsection{Validation of Transgenic Plants}

The following primers were used to validate the overexpression of transgenic plants: $F_{\text {OED }}$ (CGACACGCTTGTCTACTCCA) (targeting the $35 \mathrm{~S}$ promoter) and $\mathrm{R}_{\mathrm{OED}}$ (CGGAATTCTCAAAAGCAACTCTCTGGCAATGGGT) (targeting CDS of $C$ terminal). The following primers were used to validate RNAi transgenic plants: $F_{R D}$ (TGCAGATGCTGGCATTTAAATGTGTAA) (targeting RNAi-F fragment) and $\mathrm{R}_{\mathrm{RD}}$ (CTACGACCGGGATCCAAATACCTGCAAA) (targeting the left border of pLGNe). A 1705-bp and a 1454-bp fragment can be amplified from the OE and RNAi plants respectively; no amplification from WT. A histochemical approach was used to measure GUS activity in these transgenic plants [37,55]. The expression of CsPAE2 was then measured in transgenic plants using the $\mathrm{F}_{\mathrm{RT}}$ and $\mathrm{R}_{\mathrm{RT}}$ primers (Table S3), with WT serving as controls in all of these assays. Finally, 3 CSPAE2 overexpression plants and 3 CSPAE2 RNAi plants were obtained.

\subsection{Measurement of $C B C$ Resistance}

The resistance of transgenic plants to Xcc infection was assessed with acupuncture inoculation method as protocol in previous reports $[2,38,55]$. Briefly, 6 healthy mature leaves from each plan were obtained, and a pin $(0.5 \mathrm{~mm}$ in diameter) was used to generate 6 punctures in the surface of each leaf. A total of $1 \mu \mathrm{L}$ of an XccYN1 bacterial suspension $\left(5 \times 10^{5} \mathrm{CFU} \cdot \mathrm{mL}^{-1}\right)$ was then used to inoculate each of these pinprick spots. At $10 \mathrm{dpi}$, leaves were imaged, and Image (NIH, Bethesda, MD, USA) was used to analyze the DS and LS. The DS was calculated as in previous studies [55]. 


\section{7. $q R T-P C R$}

The frozen tissue samples were ground, and the total RNA was isolated using a miniprep kit (AidLab, Beijing, China) and then reversely transcribed to cDNA (TaKaRa, Dalin, China). Thermocycler settings of qRT-PCR were: $4 \mathrm{~min}$ at $95^{\circ} \mathrm{C} ; 40$ cycles of $95^{\circ} \mathrm{C}$ for $10 \mathrm{~s}, 56^{\circ} \mathrm{C}$ for $30 \mathrm{~s}$, and $72{ }^{\circ} \mathrm{C}$ for $30 \mathrm{~s}$. Relative expression was measured via the $2^{-\Delta \Delta C T}$ method [56]. CsActin (CAP ID: Cs1g05000, GenBank: GU911361.1) was used to normalize relative expression. Assays included three biological and three technical replicates.

\subsection{Statistical Analysis}

SPSS V22 (IBM, Chicago, IL, USA) was used for all statistical testing. The differences were evaluated using variance (ANOVA) based on Duncan's multiple range test was used to analyze the significance of differences. In the test, $p<0.05$ and $p<0.01$ were the thresholds of significance and extremely significance respectively.

Supplementary Materials: Supplementary materials can be found at http://www.mdpi.com/1422-0067/21/24/9429/ s1. Table S1. ESTs of CsPAEs. The best EST hits were extracted based on tBlastn from an EST dataset downloaded from NCBI. Table S2. The gene, CDS, and protein sequences of CsPAEs. Table S3. qRT-PCR primers used in this study. Primers were designed with the NCBI primer blast tool, with a C. sinensis mRNA database being used to check specificity.

Author Contributions: Data curation, X.Q., J.Q., and S.C.; formal analysis, Q.L. and X.Q.; funding acquisition, Z.L., S.C., and Y.H.; investigation, Q.L., J.F., W.Y., and J.Q.; project administration, Z.L., S.C., and Y.H.; writing-original draft, Q.L.; writing-review and editing, W.Y., J.Q., Z.L. and S.C. All authors have read and agreed to the published version of the manuscript.

Funding: This research was funded by the Natural Science Foundation of Chongqing (cstc2020jcyj-msxmX1064), Fundamental Research Funds for the Central Universities (XDJK2018B016), Earmarked Funds for the China Agriculture Research System (CARS-26), Opening Project of the Key Laboratory of Horticulture Science for Southern Mountainous Regions, and Key Project of Guangxi Science and Technology (GuiKeAA18118046-6).

Conflicts of Interest: The authors declare no conflict of interest.

\section{References}

1. Matzinger, P. The danger model: A renewed sense of self. Science 2002, 296, 301-305. [CrossRef] [PubMed]

2. Li, Q.; Hu, A.; Dou, W.; Qi, J.; Long, Q.; Zou, X.; Lei, T.; Yao, L.; He, Y.; Chen, S. Systematic analysis and functional validation of citrus XTH genes reveal the role of CsXTH04 in citrus bacterial canker resistance and tolerance. Front. Plant. Sci. 2019, 10, 1109. [CrossRef] [PubMed]

3. Kloth, K.J.; Abreu, I.N.; Delhomme, N.; Petř́ík, I.; Villard, C.; Ström, C.; Amini, F.; Novák, O.; Moritz, T.; Albrectsen, B.R. PECTIN ACETYLESTERASE 9 affects the transcriptome and metabolome and delays aphid feeding. Plant. Physiol. 2019, 181, 1704-1720. [CrossRef] [PubMed]

4. Yokoyama, R.; Nishitani, K. A comprehensive expression analysis of all members of a gene family encoding cell-wall enzymes allowed us to predict cis-regulatory regions involved in cell-wall construction in specific organs of Arabidopsis. Plant. Cell Physiol. 2001, 42, 1025-1033. [CrossRef] [PubMed]

5. Carpita, N.C.; Gibeaut, D.M. Structural models of primary cell walls in flowering plants: Consistency of molecular structure with the physical properties of the walls during growth. Plant. J. 1993, 3, 1-30. [CrossRef]

6. Cosgrove, D.J.; Jarvis, M.C. Comparative structure and biomechanics of plant primary and secondary cell walls. Front. Plant. Sci. 2012, 3, 204. [CrossRef]

7. Wolf, S. Plant cell wall signalling and receptor-like kinases. Biochem. J. 2017, 474, 471-492. [CrossRef]

8. Lotze, M.T.; Zeh, H.J.; Rubartelli, A.; Sparvero, L.J.; Amoscato, A.A.; Washburn, N.R.; Devera, M.E.; Liang, X.; Tör, M.; Billiar, T. The grateful dead: Damage-associated molecular pattern molecules and reduction/oxidation regulate immunity. Immunol. Rev. 2007, 220, 60-81. [CrossRef]

9. Caffall, K.H.; Mohnen, D. The structure, function, and biosynthesis of plant cell wall pectic polysaccharides. Carbohydr. Res. 2009, 344, 1879-1900. [CrossRef]

10. Harholt, J.; Suttangkakul, A.; Vibe Scheller, H. Biosynthesis of pectin. Plant. Physiol. 2010, 153, $384-395$. [CrossRef] 
11. Philippe, F.; Pelloux, J.; Rayon, C. Plant pectin acetylesterase structure and function: New insights from bioinformatic analysis. BMC Genom. 2017, 18, 456. [CrossRef] [PubMed]

12. Gou, J.Y.; Miller, L.M.; Hou, G.; Yu, X.H.; Chen, X.Y.; Liu, C.J. Acetylesterase-mediated deacetylation of pectin impairs cell elongation, pollen germination, and plant reproduction. Plant. Cell 2012, 24, 50-65. [CrossRef] [PubMed]

13. Orfila, C.; Dal Degan, F.; Jørgensen, B.; Scheller, H.V.; Ray, P.M.; Ulvskov, P. Expression of mung bean pectin acetyl esterase in potato tubers: Effect on acetylation of cell wall polymers and tuber mechanical properties. Planta 2012, 236, 185-196. [CrossRef] [PubMed]

14. Cosgrove, D.J. Plant expansins: Diversity and interactions with plant cell walls. Curr. Opin. Plant. Biol. 2015, 25, 162-172. [CrossRef]

15. Pelloux, J.; Rustérucci, C.; Mellerowicz, E.J. New insights into pectin methylesterase structure and function. Trends Plant. Sci. 2007, 12, 267-277. [CrossRef]

16. Schmidt, R.; Kunkowska, A.B.; Schippers, J.H. Role of reactive oxygen species during cell expansion in leaves. Plant. Physiol. 2016, 172, 2098-2106. [CrossRef]

17. Bethke, G.; Thao, A.; Xiong, G.; Li, B.; Soltis, N.E.; Hatsugai, N.; Hillmer, R.A.; Katagiri, F.; Kliebenstein, D.J.; Pauly, M.; et al. Pectin biosynthesis is critical for cell wall integrity and immunity in Arabidopsis thaliana. Plant. Cell 2016, 28, 537-556. [CrossRef]

18. Cantarel, B.L.; Coutinho, P.M.; Rancurel, C.; Bernard, T.; Lombard, V.; Henrissat, B. The carbohydrate-active enzymes database (CAZy): An expert resource for glycogenomics. Nucleic Acids Res. 2009, 37, D233-D238. [CrossRef]

19. De Souza, A.; Hull, P.A.; Gille, S.; Pauly, M. Identification and functional characterization of the distinct plant pectin esterases PAE8 and PAE9 and their deletion mutants. Planta 2014, 240, 1123-1138. [CrossRef]

20. De Souza, A.J.; Pauly, M. Comparative genomics of pectinacetylesterases: Insight on function and biology. Plant. Signal. Behav. 2015, 10, e1055434. [CrossRef]

21. McCarthy, T.W.; Der, J.P.; Honaas, L.A.; de Pamphilis, C.W.; Anderson, C.T. Phylogenetic analysis of pectin-related gene families in Physcomitrella patens and nine other plant species yields evolutionary insights into cell walls. BMC Plant. Biol. 2014, 14, 79. [CrossRef] [PubMed]

22. Li, Y.; Jones, L.; McQueen-Mason, S. Expansins and cell growth. Curr. Opin. Plant. Biol. 2003, 6, 603-610. [CrossRef] [PubMed]

23. Cosgrove, D.J. Assembly and enlargement of the primary cell wall in plants. Annu. Rev. Cell Dev. Biol. 1997, 13, 171-201. [CrossRef] [PubMed]

24. Randoux, B.; Renard-Merlier, D.; Mulard, G.; Rossard, S.; Duyme, F.; Sanssené, J.; Courtois, J.; Durand, R.; Reignault, P. Distinct defenses induced in wheat against powdery mildew by acetylated and nonacetylated oligogalacturonides. Phytopathology 2010, 100, 1352-1363. [CrossRef] [PubMed]

25. Chiniquy, D.; Underwood, W.; Corwin, J.; Ryan, A.; Szemenyei, H.; Lim, C.C.; Stonebloom, S.H.; Birdseye, D.S.; Vogel, J.; Kliebenstein, D.; et al. PMR5, an acetylation protein at the intersection of pectin biosynthesis and defense against fungal pathogens. Plant J. 2019, 100, 1022-1035. [CrossRef] [PubMed]

26. Pogorelko, G.; Lionetti, V.; Fursova, O.; Sundaram, R.M.; Qi, M.; Whitham, S.A.; Bogdanove, A.J.; Bellincampi, D.; Zabotina, O.A. Arabidopsis and Brachypodium distachyon transgenic plants expressing Aspergillus nidulans acetylesterases have decreased degree of polysaccharide acetylation and increased resistance to pathogens. Plant Physiol. 2013, 162, 9-23. [CrossRef]

27. Mbadinga Mbadinga, D.; Li, Q.; Ranocha, P.; Martinez, Y.; Dunand, C. Global analysis of non-animal peroxidases provides insights into the evolution of this gene family in the green lineage. J. Exp. Bot. 2020, 71, 3350-3360. [CrossRef]

28. De Palma, M.; Salzano, M.; Villano, C.; Aversano, R.; Lorito, M.; Ruocco, M.; Docimo, T.; Piccinelli, A.L.; D'Agostino, N.; Tucci, M. Transcriptome reprogramming, epigenetic modifications and alternative splicing orchestrate the tomato root response to the beneficial fungus. Hortic. Res. 2019, 6, 5. [CrossRef]

29. Ferrari, S.; Galletti, R.; Pontiggia, D.; Manfredini, C.; Lionetti, V.; Bellincampi, D.; Cervone, F.; De Lorenzo, G. Transgenic expression of a fungal endo-polygalacturonase increases plant resistance to pathogens and reduces auxin sensitivity. Plant Physiol. 2008, 146, 669-681. [CrossRef]

30. Li, Q.; Qi, J.; Qin, X.; Dou, W.; Dunand, C.; Chen, S.; He, Y. CsPrx25, a class III peroxidase in Citrus sinensis, confers resistance to citrus bacterial canker through the maintenance of ROS homoeostasis and cell wall lignification. Hortic. Res. 2020, 7, 142. [CrossRef] 
31. Li, Q.; Hu, A.H.; Qi, J.J.; Dou, W.F.; Qin, X.J.; Zou, X.P.; Xu, L.Z.; Chen, S.C.; He, Y.R. CsWAKL08, a pathogen-induced wall-associated receptor-like kinase in sweet orange, confers resistance to citrus bacterial canker via ROS control and JA signaling. Hortic. Res. 2020, 7, 15. [CrossRef]

32. Li, Q.; Dou, W.F.; Qi, J.J.; Qin, X.J.; Chen, S.C.; He, Y.R. Genomewide analysis of the CIII peroxidase family in sweet orange (Citrus sinensis) and expression profiles induced by Xanthomonas citri subsp. citri and hormones. J. Genet. 2020, 99, 13. [CrossRef]

33. Schaad, N.W.; Postnikova, E.; Lacy, G.H.; Sechler, A.; Agarkova, I.; Stromberg, P.E.; Stromberg, V.K.; Vidaver, A.K. Emended classification of xanthomonad pathogens on citrus. Syst. Appl. Microbiol. 2005, 28, 494-518. [CrossRef] [PubMed]

34. Xu, Q.; Chen, L.L.; Ruan, X.; Chen, D.; Zhu, A.; Chen, C.; Bertrand, D.; Jiao, W.B.; Hao, B.H.; Lyon, M.P.; et al. The draft genome of sweet orange (Citrus sinensis). Nat. Genet. 2013, 45, 59-66. [CrossRef] [PubMed]

35. Wang, J.; Chen, D.; Lei, Y.; Chang, J.W.; Hao, B.H.; Xing, F.; Li, S.; Xu, Q.; Deng, X.X.; Chen, L.L. Citrus sinensis annotation project (CAP): A comprehensive database for sweet orange genome. PLoS ONE 2014, 9, e87723. [CrossRef] [PubMed]

36. Li, Q.; Yu, H.; Cao, P.B.; Fawal, N.; Mathé, C.; Azar, S.; Cassan-Wang, H.; Myburg, A.A.; Grima-Pettenati, J.; Marque, C.; et al. Explosive tandem and segmental duplications of multigenic families in Eucalyptus grandis. Genome Biol. Evol. 2015, 7, 1068-1081. [CrossRef]

37. Sendín, L.N.; Orce, I.G.; Gómez, R.L.; Enrique, R.; Grellet Bournonville, C.F.; Noguera, A.S.; Vojnov, A.A.; Marano, M.R.; Castagnaro, A.P.; Filippone, M.P. Inducible expression of Bs2 R gene from Capsicum chacoense in sweet orange (Citrus sinensis L. Osbeck) confers enhanced resistance to citrus canker disease. Plant. Mol. Biol. 2017, 93, 607-621. [CrossRef]

38. Peng, A.; Chen, S.; Lei, T.; Xu, L.; He, Y.; Wu, L.; Yao, L.; Zou, X. Engineering canker-resistant plants through CRISPR/Cas9-targeted editing of the susceptibility gene CsLOB1 promoter in citrus. Plant. Biotechnol. J. 2017, 15, 1509-1519. [CrossRef]

39. Li, Q.; Jia, R.; Dou, W.; Qi, J.; Qin, X.; Fu, Y.; He, Y.; Chen, S. CsBZIP40, a BZIP transcription factor in sweet orange, plays a positive regulatory role in citrus bacterial canker response and tolerance. PLOS ONE 2019, 14, e0223498. [CrossRef]

40. Morello, L.; Breviario, D. Plant spliceosomal introns: Not only cut and paste. Curr. Genom. 2008, 9, $227-238$. [CrossRef]

41. Song, H.; Wang, P.; Lin, J.Y.; Zhao, C.; Bi, Y.; Wang, X. Genome-wide identification and characterization of WRKY gene family in peanut. Front. Plant. Sci. 2016, 7, 534. [CrossRef] [PubMed]

42. Goodstein, D.M.; Shu, S.; Howson, R.; Neupane, R.; Hayes, R.D.; Fazo, J.; Mitros, T.; Dirks, W.; Hellsten, U.; Putnam, N.; et al. Phytozome: A comparative platform for green plant genomics. Nucleic Acids Res. 2012, 40, D1178-D1186. [CrossRef] [PubMed]

43. Wu, G.A.; Prochnik, S.; Jenkins, J.; Salse, J.; Hellsten, U.; Murat, F.; Perrier, X.; Ruiz, M.; Scalabrin, S.; Terol, J.; et al. Sequencing of diverse mandarin, pummelo and orange genomes reveals complex history of admixture during citrus domestication. Nat. Biotechnol. 2014, 32, 656-662. [CrossRef] [PubMed]

44. Fawal, N.; Li, Q.; Mathé, C.; Dunand, C. Automatic multigenic family annotation: Risks and solutions. Trends Genet. 2014, 30, 323-325. [CrossRef] [PubMed]

45. Potter, S.C.; Luciani, A.; Eddy, S.R.; Park, Y.; Lopez, R.; Finn, R.D. HMMER web server: 2018 update. Nucleic Acids Res. 2018, 46, W200-W204. [CrossRef] [PubMed]

46. Prakash, A.; Jeffryes, M.; Bateman, A.; Finn, R.D. The HMMER web server for protein sequence similarity search. Curr. Protoc. Bioinform. 2017, 60, 13.15.1-13.15.23. [CrossRef]

47. Letunic, I.; Bork, P. 20 years of the SMART protein domain annotation resource. Nucleic Acids Res. 2018, 46, D493-D496. [CrossRef]

48. Li, Q.; Qi, J.; Qin, X.; Dou, W.; Lei, T.; Hu, A.; Jia, R.; Jiang, G.; Zou, X.; Long, Q.; et al. CitGVD: A comprehensive database of citrus genomic variations. Hortic. Res. 2020, 7, 12. [CrossRef]

49. Kumar, S.; Stecher, G.; Tamura, K. MEGA7: Molecular evolutionary genetics analysis version 7.0 for bigger datasets. Mol. Biol. Evol. 2016, 33, 1870-1874. [CrossRef]

50. Hu, B.; Jin, J.; Guo, A.Y.; Zhang, H.; Luo, J.; Gao, G. GSDS 2.0: An upgraded gene feature visualization server. Bioinformatics 2015, 31, 1296-1297. [CrossRef]

51. Voorrips, R.E. MapChart: Software for the graphical presentation of linkage maps and QTLs. J. Hered 2002, 93, 77-78. [CrossRef] [PubMed] 
52. Petersen, T.N.; Brunak, S.; von Heijne, G.; Nielsen, H. SignalP 4.0: Discriminating signal peptides from transmembrane regions. Nat. Methods 2011, 8, 785-786. [CrossRef] [PubMed]

53. Yu, C.S.; Chen, Y.C.; Lu, C.H.; Hwang, J.K. Prediction of protein subcellular localization. Proteins 2006, 64, 643-651. [CrossRef] [PubMed]

54. Li, Q.; Qi, J.; Qin, X.; Hu, A.; Fu, Y.; Chen, S.; He, Y. Systematic identification of lysin-motif receptor-like kinases (LYKs) in Citrus sinensis, and analysis of their inducible involvements in citrus bacterial canker and phytohormone signaling. Sci. Hortic. 2021, 276. [CrossRef]

55. He, Y.; Jia, R.; Qi, J.; Chen, S.; Lei, T.; Xu, L.; Peng, A.; Yao, L.; Long, Q.; Li, Z.; et al. Functional analysis of citrus AP2 transcription factors identified CsAP2-09 involved in citrus canker disease response and tolerance. Gene 2019, 707, 178-188. [CrossRef]

56. Livak, K.J.; Schmittgen, T.D. Analysis of relative gene expression data using real-time quantitative PCR and the 2(-delta delta C(T)) method. Methods 2001, 25, 402-408. [CrossRef]

Publisher's Note: MDPI stays neutral with regard to jurisdictional claims in published maps and institutional affiliations.

(C) 2020 by the authors. Licensee MDPI, Basel, Switzerland. This article is an open access article distributed under the terms and conditions of the Creative Commons Attribution (CC BY) license (http://creativecommons.org/licenses/by/4.0/). 